\title{
Populus trichocarpa and Populus deltoides Exhibit Different Metabolomic Responses to Colonization by the Symbiotic Fungus Laccaria bicolor
}

\author{
Timothy J. Tschaplinski, ${ }^{1}$ Jonathan M. Plett, ${ }^{2,3}$ Nancy L. Engle, ${ }^{1}$ Aurelie Deveau, ${ }^{2}$ Katherine C. Cushman, ${ }^{1}$ \\ Madhavi Z. Martin, ${ }^{1}$ Mitchel J. Doktycz, ${ }^{1}$ Gerald A. Tuskan,, ${ }^{1}$ Annick Brun, ${ }^{2}$ Annegret Kohler, ${ }^{2}$ and \\ Francis Martin² \\ ${ }^{1}$ Plant Systems Biology Group, Biosciences Division, Oak Ridge National Laboratory, Oak Ridge, TN 37831-6445, U.S.A.; \\ ${ }^{2}$ UMR INRA-UHP 1136 Interactions Arbres/Micro-organismes, Centre INRA de Nancy, Champenoux 54280, France; \\ ${ }^{3}$ Hawkesbury Institute for the Environment, University of Western Sydney, Richmond, NSW 2753, Australia
}

Submitted 27 September 2013. Accepted 4 February 2014.

\begin{abstract}
Within boreal and temperate forest ecosystems, the majority of trees and shrubs form beneficial relationships with mutualistic ectomycorrhizal (ECM) fungi that support plant health through increased access to nutrients as well as aiding in stress and pest tolerance. The intimate interaction between fungal hyphae and plant roots results in a new symbiotic "organ" called the ECM root tip. Little is understood concerning the metabolic reprogramming that favors the formation of this hybrid tissue in compatible interactions and what prevents the formation of ECM root tips in incompatible interactions. We show here that the metabolic changes during favorable colonization between the ECM fungus Laccaria bicolor and its compatible host, Populus trichocarpa, are characterized by shifts in aromatic acid, organic acid, and fatty acid metabolism. We demonstrate that this extensive metabolic reprogramming is repressed in incompatible interactions and that more defensive compounds are produced or retained. We also demonstrate that $L$. bicolor can metabolize a number of secreted defensive compounds and that the degradation of some of these compounds produces immune response metabolites (e.g., salicylic acid from salicin). Therefore, our results suggest that the metabolic responsiveness of plant roots to $L$. bicolor is a determinant factor in fungus-host interactions.
\end{abstract}

Plants are constantly in contact with a wide range of microorganisms. As such, plants have evolved a complex signaling network that alters the biochemistry and physical properties of plant tissues to create an inhospitable environment for the invading organism (Berger et al. 2007). Despite such defenses, some organisms are able to tolerate, bypass, or modulate the response of the plant, leading to colonization of plant tissues and eventual access to nutrients stored within the plant. One

The complete expression dataset is available in the Gene Expression Omnibus database (accession number GSE54789) at the National Center for Biotechnology Information.

Corresponding author: J. M. Plett; E-mail: j.plett@uws.edu.au

* The $\boldsymbol{e}$-Xtra logo stands for "electronic extra" and indicates that a supplementary table is published online.

(C) 2014 The American Phytopathological Society class of organisms that can so manipulate plants are found within the group of mutualistic microbes called the ectomycorrhizal (ECM) fungi, found ubiquitously in forest soils worldwide. Invasive colonization by this group of fungi leads to pronounced morphological changes of both the root and fungal cell architecture and results in the creation of a new hybrid organ called the ECM root tip (Burgess et al. 1996; Felten et al. 2009; Kottke and Oberwinkler 1987). In these modified root tips, fungal hyphae are found surrounding the root's surface, forming a fungal sheath called the mantle, as well as within the root apoplastic space, where these hyphae form a complex mixture of plant and fungal cells called the Hartig net (Gea et al. 1994). Hyphae within functioning ECM root tips never enter the plant cell but, rather, exchange nutrients across the large surface area of the plant-fungus interface within the Hartig net. Many studies have sought to predict the alterations to plant and fungal metabolism during the colonization of a receptive host (Ceccaroli et al. 2011; Deveau et al. 2008; Larsen et al. 2011; Nehls et al. 2001; Reich et al. 2009). Although fewer studies have characterized the actual metabolic shifts in these specialized organs, it is known that colonization of plant tissues by an ECM fungus results in an increase of sucrose catabolism (Martin et al. 1998; Schaeffer et al. 1995), an increase in some free amino acids (e.g., Glx, Ala, and Cys), and a decrease in others (e.g., Arg, Met, and Ser) (Finlay et al. 1988, 1989), whereas fatty acid profiles have not been shown to change (Luo et al. 2009).

Although we have a basic overview of how ECM fungi may affect the host plant metabolome, very little is known concerning how the host metabolome shifts over the course of the colonization process and, further, what differentiates the metabolism found within a compatible mutualist-plant host interaction as opposed to an incompatible interaction. We sought to address this lack of knowledge in a two-pronged experiment. We first profiled the metabolome of the compatible interaction between the mutualistic ECM fungus Laccaria bicolor and its natural host, Populus trichocarpa. L. bicolor was chosen due to the genomic and transcriptomic data currently available (Larsen et al. 2011; Martin et al. 2008). We then contrasted these results to two different incompatible relationships: i) where wild-type (WT) L. bicolor attempts to colonize a recalcitrant host (e.g., P. deltoides) (Labbé et al. 2011) and ii) by using a mutated version of $L$. bicolor that is unable to colonize its natural host, P. trichocarpa. We demonstrate here that compatible hostmicrobe interactions are characterized by a lower level of 
certain defense-related secondary metabolites compared with incompatible interactions and that compatible interactions lead to a more dynamic metabolic response in the symbiotic tissues over the course of colonization. This latter observation is potentially due to the increased presence of active fungal tissues in roots in compatible interactions compared with incompatible interactions. Further, our results demonstrate that $\mathrm{L}$. bicolor is able to metabolize certain secondary metabolites present in roots undergoing colonization. Therefore, our results would suggest that, whereas the mechanism controlling hostmicrobe compatibility are similar in both pathogenic and mutualistic interactions, there are also distinct differences with regard to the regulation of aromatic acids, organic acids, and fatty acid metabolism.

\section{RESULTS}

\section{L. bicolor colonizes the roots of multiple Populus spp.}

We chose to study the different stages of colonization of newly rooted $P$. trichocarpa saplings by the model mutualistic ECM fungus L. bicolor (WT) after 2, 4, 6, and 12 weeks of contact, and determined both macroscopically and microscopically the extent of colonization of the root system. The first colonized roots became macroscopically visible at 2 weeks of contact, the number of which increased steadily through 12 weeks of contact (Fig. 1A). Each stage of colonization was marked by a commensurate increase in the amount of fungal tissue integrated into the mycorrhizal root as determined by microscopic analysis of roots in contact with L. bicolor (WT). At 2 weeks of contact, a loose mantle had begun to form around young lateral roots and begun to grow in between the cells of the rhizoderm. By 4 and 6 weeks of colonization, the mantle had formed and a Hartig net had begun to develop, with maximal colonization achieved after 12 weeks of contact (Fig. 1A). In order to compare the effect of the host plant on the ability of L. bicolor to colonize roots, we compared the mycorrhization potential and morphology of mycorrhizal root tips of $P$. trichocarpa to another poplar species, $P$. deltoides. It was found that, compared with $P$. trichocarpa (44\% of lateral roots colonized after 12 weeks of contact), $P$. deltoides had a significantly lower rate of colonization $(12 \%$ of lateral roots colonized) and a significantly less-developed Hartig net (Fig. 1B). These results confirm previous results that have demonstrated the differential ability of Laccaria spp. to colonize different host genera (e.g., Pseudotsuga) (Molina 1982) and different species within a specific host genus (e.g., Populus spp.) (Labbé et al. 2011). We compared these results to the ability of a mutant version of $L$. bicolor (lacking production of the MiSSP7 protein) (Plett et al. 2011) to colonize P. trichocarpa. We found that, as in the L. bicolor (WT)-P. deltoides association, the colonization ability of three independent transgenic lines of L. bicolor missp 7 mutants (CL03, CL09, and CL19) was significantly reduced and that Hartig net development was almost completely abolished (Fig. 1B). Therefore, the L. bicolor (WT)-P. trichocarpa association is an example of a compatible interaction and the $L$. bicolor (WT)-P. deltoides and L. bicolor missp7-P. trichocarpa associations are examples of incompatible relationships.

\section{Evolution of the symbiotic metabolome in the compatible $L$. bicolor-P. trichocarpa interaction.}

Mycorrhizal colonization of P. trichocarpa fine roots by $L$. bicolor is associated with rapid and pronounced changes in the combined metabolome (Table 1). After 2 weeks of contact between L. bicolor and P. trichocarpa, of the 105 metabolites quantified, 9 were significantly regulated. At this time point, an unidentified alkaloid (retention time followed by key mass-to- charge [m/z] ratios; $10.10 \mathrm{~m} / \mathrm{z} 346$ 244) was only found in roots exhibiting the initial stages of colonization (Fig. 1; Table 1). Arbutin (hydroquinone glucoside), a poplar metabolite that is typically present in low concentration, was the next most responsive metabolite, exhibiting a 12-fold increase in concentration (Table 1). There was also a large and early accumulation of trehalose, a known fungal storage carbohydrate, in mycorrhizal $P$. trichocarpa roots. Other early increased responses in $P$. trichocarpa
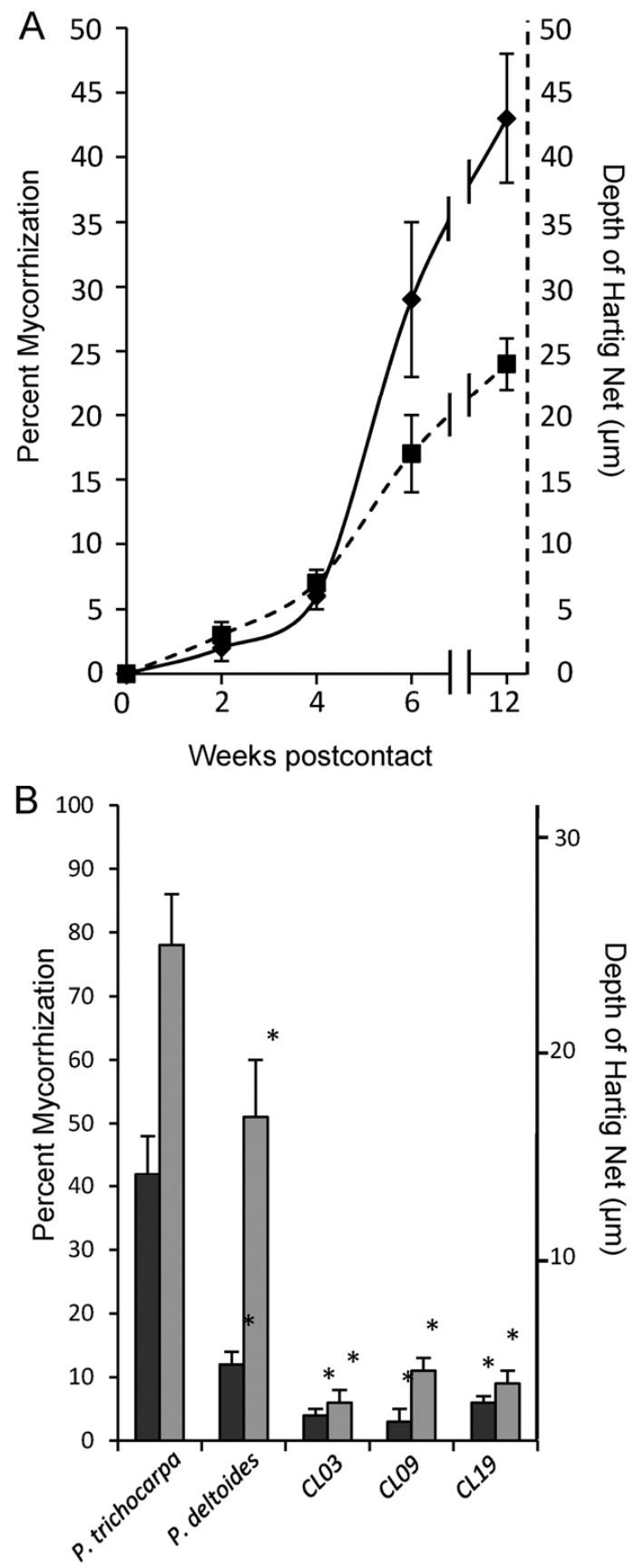

Fig. 1. Laccaria bicolor exhibits different abilities to colonize the roots of different Populus spp. A, L. bicolor colonizes the root system of P. trichocarpa gradually over a 12 -week period, with both the number of colonized roots increasing steadily after 2 weeks of contact between the fungus and the root system (solid line) as well as the depth of the Hartig net within the root apoplast of colonized roots (dashed line). B, P. deltoides shows a lower number of colonized lateral roots compared with $P$. trichocarpa (dark-gray bars and left $x$ axis), as do three transgenic lines of L. bicolor missp7 (Cl03, C109, and C119) colonizing P. trichocarpa. Hartig net depth (light-gray bars and right $x$ axis) is also reduced. Asterisk $(*)=P<0.05$; \pm standard error. 
Table 1. Metabolic responses associated with establishment of the Populus-Laccaria symbiotic relationship ${ }^{\mathrm{a}}$

\begin{tabular}{|c|c|c|c|c|}
\hline \multirow[b]{2}{*}{ Metabolite (RT-m/z) } & \multicolumn{4}{|c|}{ Mycorrhizal versus control roots fold-change } \\
\hline & 2 Weeks & 4 Weeks & 6 Weeks & 12 Weeks \\
\hline 10.10244346 Alkaloid & $\infty *$ & $\infty$ & $\infty$ & $\infty *$ \\
\hline 10.19230215217 & $0.2 *$ & $0.2^{*}$ & $0.3^{*}$ & 2.4 \\
\hline 10.91244349259217 & 1.2 & $7.5^{*}$ & $3.7 *$ & $10.1^{*}$ \\
\hline 11.02217450 Dehydro sugar & 0.6 & 0.5 & $1.5^{*}$ & $2.0^{*}$ \\
\hline 11.02235 Coumaroyl glycoside & 1.6 & 2.0 & $3.9 *$ & $10.1 *$ \\
\hline 11.10393303257 & 0.5 & $0.1 *$ & 0.1 & 0.0 \\
\hline 12.56495585 Glycoside & $0.0^{*}$ & $0.0^{*}$ & $0.0^{*}$ & 0.0 \\
\hline 13.97 Benzyl glycoside & $0.4 *$ & 0.6 & 2.5 & 1.1 \\
\hline 14.65 319 Mannitol conjugate & 0.6 & 0.2 & $0.7 *$ & 1.0 \\
\hline 15.14314 Glycoside & 0.4 & 0.5 & 1.3 & 1.6 \\
\hline 15.21361292375 & 0.6 & 0.1 & $0.6^{*}$ & 0.7 \\
\hline 15.90297 Guaiacyl lignan & 0.6 & 0.3 & $0.8^{*}$ & 1.6 \\
\hline 25.67648 Kaempferol conjugate & 0.1 & 0.1 & 0.2 & 0.1 \\
\hline 6.17134239184 & 0.5 & 6.1 & $3.3^{*}$ & $16.8^{*}$ \\
\hline 6.99207252237133 & 0.5 & $5.3^{*}$ & $3.2 *$ & $18.2 *$ \\
\hline 7.84157 & 0.4 & 22.2 & 3.2 & $83.6^{*}$ \\
\hline 7.89211 & 0.6 & $4.1 *$ & $3.4 *$ & $22.3^{*}$ \\
\hline 8.7599155169195 & 0.8 & 0.4 & $2.9 *$ & $10.2 *$ \\
\hline 8.96239253199170 & 1.5 & $16.1 *$ & $4.3 *$ & $14.4 *$ \\
\hline Aconitic acid & 0.4 & 0.1 & $0.5^{*}$ & 1.7 \\
\hline 1,6-Anhydroglucose & 0.7 & 0.3 & 1.3 & 1.9 \\
\hline Arbutin & $12.2^{*}$ & $38.7 *$ & $90.3 *$ & $386.9 *$ \\
\hline Aspartic acid & 0.4 & 0.6 & $1.7^{*}$ & 3.0 \\
\hline Benzoic acid & 1.7 & 0.7 & 3.1 & $5.5^{*}$ \\
\hline 1-O-Caffeoylquinic acid & 0.1 & 0.7 & 0.8 & $0.3 *$ \\
\hline c-3-O-Caffeoylquinic acid & 0.5 & $0.4^{*}$ & 0.3 & 0.3 \\
\hline t-3-O-Caffeoylquinic acid & 0.2 & 0.1 & $0.2^{*}$ & 0.2 \\
\hline 5-O-Caffeoylshikimate & 0.3 & 0.6 & 0.6 & 0.1 \\
\hline (+)-Catechin & 0.2 & $0.4^{*}$ & 0.6 & 0.3 \\
\hline Catechol & 0.8 & 3.1 & $2.7 *$ & $4.7 *$ \\
\hline Citric acid & 0.1 & 0.0 & $0.1 *$ & $0.2 *$ \\
\hline 3-Hydroxybenzoic acid & 0.6 & 1.3 & $2.9 *$ & $7.2 *$ \\
\hline 4-Hydroxybenzoic acid & 0.8 & 1.0 & $1.6^{*}$ & $4.2 *$ \\
\hline 2,5-Dihydroxybenzoic acid & 0.8 & 0.7 & $3.3 *$ & $3.4^{*}$ \\
\hline 3,4-Dihydroxybenzoic acid & 0.3 & 1.7 & 0.9 & 4.4 \\
\hline Ethyl-phosphate & $0.3 *$ & 0.7 & $0.5 *$ & 0.3 \\
\hline Fructose & 0.3 & 0.2 & 1.6 & 0.3 \\
\hline Fumaric acid & 0.8 & 1.3 & $2.4 *$ & 2.5 \\
\hline Galactose & 0.4 & 0.8 & 1.1 & 1.1 \\
\hline Galactosylpyranosylglycerol & 0.4 & 0.4 & $0.4 *$ & 1.3 \\
\hline Glucose & 0.3 & 0.3 & 0.5 & 0.5 \\
\hline Glutamic acid & $0.3^{*}$ & 0.7 & 0.8 & 3.4 \\
\hline Glycerol & 0.9 & 0.5 & $4.2 *$ & $3.2 *$ \\
\hline Grandidentatin & 0.4 & 0.3 & 0.7 & 1.7 \\
\hline Hydroquinone & 0.8 & 2.3 & $8.8^{*}$ & $97.9 *$ \\
\hline Hydroxyquinol & 0.6 & 2.4 & $1.7 *$ & 6.3 \\
\hline$\alpha$-Linolenic acid & 0.6 & $2.7 *$ & 1.1 & 2.0 \\
\hline Maleic acid & 0.2 & 0.6 & 1.0 & 0.7 \\
\hline Malic acid & 0.4 & 0.1 & 0.7 & 0.2 \\
\hline Myoinositol & $2.3 *$ & 0.3 & $0.4 *$ & 0.2 \\
\hline Myoinositol-2P & 0.4 & $1.9^{*}$ & 1.1 & 1.5 \\
\hline Oxalomalic acid & $2.9 *$ & 0.4 & $3.5^{*}$ & 1.1 \\
\hline Palmitic acid & 1.0 & $4.8^{*}$ & $2.5^{*}$ & $5.9 *$ \\
\hline Phosphate & 0.4 & 0.3 & 0.7 & 1.2 \\
\hline 5-oxo-Proline & 0.3 & 1.5 & 1.7 & 2.4 \\
\hline Pyruvic acid & 1.1 & 1.7 & $2.9 *$ & $6.6^{*}$ \\
\hline Quinic acid & 0.2 & 0.1 & $0.2 *$ & 0.1 \\
\hline Salicin & 0.6 & 1.8 & 1.1 & 0.6 \\
\hline Salicortin & 0.6 & 1.6 & 1.1 & 0.1 \\
\hline Salicylic acid & 0.7 & 1.1 & 1.8 & 0.8 \\
\hline$\alpha$-Salicyloylsalicin & 1.5 & 1.8 & 0.9 & 0.3 \\
\hline Shikimic acid & 0.4 & $0.4^{*}$ & $0.5^{*}$ & 0.2 \\
\hline$\beta$-Sitosterol & 0.4 & $4.1^{*}$ & 1.0 & 1.9 \\
\hline Stearic acid & 0.9 & $5.5^{*}$ & $6.0^{*}$ & 8.9 \\
\hline Stigmasterol & 0.6 & $4.6^{*}$ & 1.0 & 3.8 \\
\hline Succinic acid & 0.7 & 1.0 & $2.1 *$ & $2.7 *$ \\
\hline Sucrose & 0.7 & 0.1 & 0.7 & 0.3 \\
\hline Syringic acid & 0.9 & 2.8 & $2.5^{*}$ & $6.4 *$ \\
\hline Threonic acid & 0.6 & 0.8 & $4.5^{*}$ & 4.9 \\
\hline Trehalose & 21.7 & $12.7 *$ & $55.0^{*}$ & $39.7 *$ \\
\hline Trichocarpin & 3.2 & 0.2 & 2.3 & $0.3^{*}$ \\
\hline 1,3,5-Trihydroxybenzene & 0.6 & 0.8 & $2.0 *$ & $3.1 *$ \\
\hline Tryptophan & 0.3 & 0.1 & 1.1 & 0.4 \\
\hline
\end{tabular}


included increases in oxalomalic acid and myoinositol; however, these were short-lived responses (Table 1).

After 4 weeks of contact between L. bicolor and P. trichocarpa, of the 105 metabolites quantified, 18 metabolites were significantly regulated. The large majority of significantly regulated metabolites were found not to be regulated at the earlier time point. These metabolites included a number of upregulated unidentified compounds $(8.96 \mathrm{~m} / \mathrm{z} 239253199 \quad 170$, $10.91 \mathrm{~m} / \mathrm{z} 244349259217$, and $6.99 \mathrm{~m} / \mathrm{z} 207252237133$ ) (Table 1) as well as stearic acid, palmitic acid, stigmasterol, $\alpha$ linolenic acid, and $\beta$-sitosterol. We also found that levels of arbutin continued to rise above their 2 -week levels. Of those metabolites with reduced levels at this time point were (+)-catechin, c-3-O-caffeoylquinic acid, and shikimic acid as well as a number of unknowns (e.g., $12.56 \mathrm{~m} / \mathrm{z} 495585$ glycoside) (Table 1). Major sugars, including glucose, fructose, and sucrose, tended to be reduced as early as 4 weeks $(P<0.1)$ (Table 1) but concentrations were generally variable over the course of the study.

The largest number of significantly regulated metabolites was found at the 6 -week point of colonization, with 41 metabolites differentially regulated over control tissues (Table 1). Although the majority of the metabolites found to accumulate at significantly higher levels after 4 weeks of colonization were also found at this time point, we also identified a range of metabolites not previously found to have been significantly regulated (Table 1). A number of fatty acids and other metabolites related to lipid metabolism increased at these time points. These included stearic acid, palmitic acid, $\alpha$-linolenic acid, glycerol, and $\beta$-sitosterol, all metabolites common to both organisms. The increases in such metabolites are in contrast to declines observed in several other metabolites common to both organisms, as the plant-fungus association was established.
Significant declines were evident for galactopyranosyl glycerol, partially identified lignins (aromatic metabolites), and organic acids related to secondary metabolism, including shikimic acid and quinic acid, and some citric acid (trichloroacetic acid [TCA]) cycle organic acids, including citric acid and aconitic acid, which contrasted markedly with TCA cycle organic acids that increased, including fumaric acid and succinic acid (Table 1 ).

After week 12, the point at which ECM root tips were mature and functional, we identified 27 metabolites that were significantly regulated. There were very few metabolites that were found to be uniquely regulated at this stage of the interaction between L. bicolor and P. trichocarpa. As in previous time points, the most highly accumulated metabolites (in addition to the unidentified alkaloid $10.10 \mathrm{~m} / \mathrm{z} 244$ 346) were arbutin and hydroquinone. Additionally, catechol, another dihydroxybenzene metabolite that is readily detectable in poplar, accumulated five- to sevenfold by week 12 . The symbiotic sugar storage molecule trehalose was also increased significantly in fold-change at this stage, with an 83-fold increase over control tissues. In contrast, there was also an initial decline in glutamic acid and sustained reductions in an atypical metabolite, ethyl-phosphate, and a number of unidentified metabolites, such as $12.56 \mathrm{~m} / \mathrm{z} 495585$ glycoside, and $11.10 \mathrm{~m} / \mathrm{z}$ 393, 303 257 (by week 4), which were all reduced to negligible levels over time.

\section{L. bicolor colonization increases flux into the benzoate degradation pathway.}

Throughout the colonization process of $P$. trichocarpa roots, the metabolites that exhibited the most consistent and sustained rise in concentration were arbutin and hydroquinone. The significant increase in the aromatic metabolite arbutin, coupled

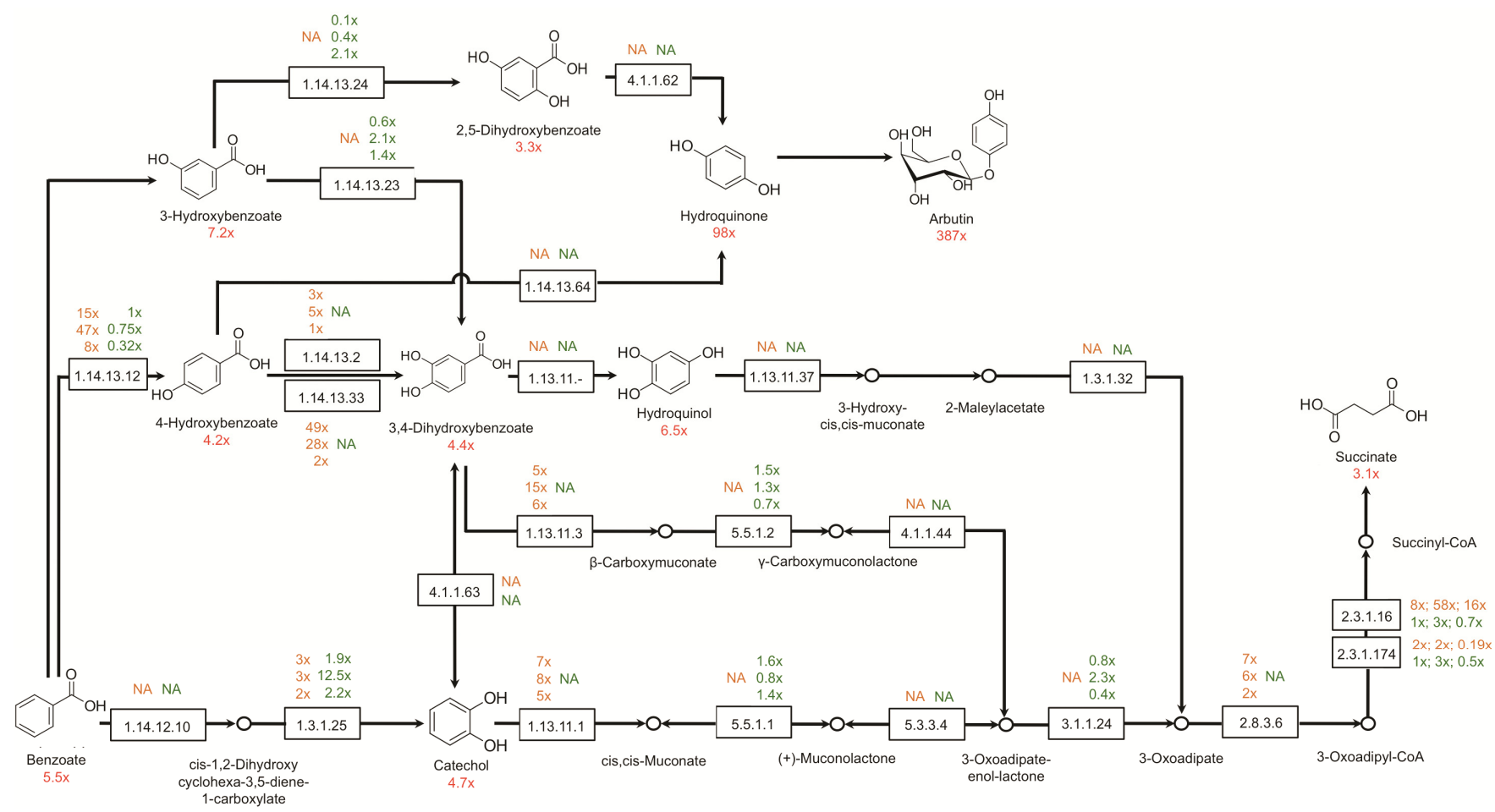

Fig. 2. Regulation of the benzoate degradation pathway during colonization of Populus trichocarpa root tissues by Laccaria bicolor. Benzoate degradation pathway (adapted from the reference pathway as curated by the Kyoto Encyclopedia of Genes and Genomes) showing accumulation of arbutin and its precursors as demonstrated by metabolomic analysis. Structures of quantified metabolites are shown. Values in red beneath structures indicate fold-change in metabolites measured in P. trichocarpa roots colonized by L. bicolor after 12 weeks of contact as compared with uncolonized roots. Values above enzyme numbers indicate fold-change in transcript abundance of the closest homologue to each enzyme in $L$. bicolor (brown values) and in $P$. trichocarpa (green values) in colonized tissues versus uncolonized tissues after 2, 4, and 12 weeks of contact between the two organisms. Expression levels were determined using RNA-Seq. Values marked "NA" indicate enzymes that are "not annotated" in the genomes. 
Table 2. Metabolite fold-change responses in the ectomycorrhizal root tips of Laccaria bicolor (wild type [WT])- Populus trichocarpa compared with L. bicolor (WT)-P. deltoides or L. bicolor missp7-P. trichocarpa (CL03, CL09, and CL19) compared with control tissues after 12 weeks of contact $^{\mathrm{a}}$

\begin{tabular}{|c|c|c|c|c|c|}
\hline \multirow[b]{2}{*}{ Metabolite (RT-m/z) } & \multicolumn{5}{|c|}{ Mycorrhizal versus control tissues fold-change } \\
\hline & P. trichocarpa & P. deltoides & CLO9 & CL19 & CL03 \\
\hline 10.12244318346 Alkaloid & $\infty *$ & $\infty *$ & $\infty *$ & $\infty *$ & $\infty *$ \\
\hline 10.91244349259217 & $10.1 *$ & $4.3 *$ & 0.6 & 0.5 & 0.8 \\
\hline 10.98141288347251 & $2.4^{*}$ & ND & 0.7 & 0.6 & 0.9 \\
\hline 11.02217450 Dehydro sugar & $2.0^{*}$ & 1.9 & $0.6 *$ & 0.9 & 1 \\
\hline 11.02235 Coumaroyl glycoside & $10.1^{*}$ & $4.4^{*}$ & 0.9 & 0.8 & 1.0 \\
\hline 11.10338397366217 & $2.6^{*}$ & ND & 0.7 & 0.7 & 0.9 \\
\hline 11.18397170155 & $2.5^{*}$ & $7.1 *$ & 0.9 & 0.9 & 1.0 \\
\hline 12.05244397412413 & $3.1^{*}$ & ND & 0.8 & 0.6 & 1.0 \\
\hline 12.28 254117 Hydroquinone-conjugate & 0.5 & ND & $0.3 *$ & $0.3 *$ & 0.4 \\
\hline 12.32258278 & $3.1^{*}$ & ND & 0.9 & 0.8 & 1.0 \\
\hline 12.52278265 Glycoside & 0.6 & 0.5 & $0.3 *$ & $0.4 *$ & $0.3 *$ \\
\hline 13.00383346 & $3.1 *$ & ND & 0.8 & 0.7 & 1.0 \\
\hline 13.05193262 & $2.9 *$ & ND & 0.8 & 0.5 & 1.1 \\
\hline 13.51641319306293 & $1.9 *$ & 0.5 & $0.6^{*}$ & 0.7 & 1.3 \\
\hline 14.63254424409362 & $\infty *$ & ND & $\infty *$ & $\infty *$ & $\infty$ \\
\hline 14.93254342 Glycoside & $\infty *$ & ND & $\infty *$ & $\infty *$ & $\infty$ \\
\hline 14.98254291 Glycoside & $\infty *$ & ND & $\infty *$ & $\infty *$ & $\infty$ \\
\hline 15.21 437 Glycoside & $0.6^{*}$ & ND & $0.2 *$ & $0.2 *$ & 0.5 \\
\hline 25.78648 Kaempferol conjugate & 1.0 & 0.1 & 0.5 & 0.5 & 1.2 \\
\hline 6.99207252237133 & $18.2 *$ & $9.0 *$ & $0.3 *$ & $0.2 *$ & 0.6 \\
\hline 7.84157 & $83.6^{*}$ & $17.8^{*}$ & 0.4 & 1.1 & 0.7 \\
\hline 7.89211 & $22.3^{*}$ & $12.1 *$ & 0.5 & 0.5 & 0.6 \\
\hline 8.33172114274289 & $2.7 *$ & ND & 0.8 & 0.4 & 0.9 \\
\hline 8.7599155169195 & $10.2 *$ & $4.1 *$ & 0.6 & 0.6 & 0.9 \\
\hline 8.96239253199170 & $14.4 *$ & $4.0^{*}$ & $0.6^{*}$ & 0.5 & 0.8 \\
\hline 9.34254239174 & $3.3^{*}$ & ND & 0.9 & 1.0 & 1.1 \\
\hline 9.43254219187172 & $2.4 *$ & $5.5^{*}$ & 0.7 & 0.7 & 0.9 \\
\hline 9.67191145 & $2.2 *$ & $3.6^{*}$ & 0.6 & 0.6 & 0.9 \\
\hline 9.70193244329309 & $2.5^{*}$ & ND & 0.7 & 0.8 & 0.9 \\
\hline Arbutin & $386.9 *$ & $29.9 *$ & 14.1 & 17.2 & 17.2 \\
\hline Benzoic acid & $5.5^{*}$ & $4.2^{*}$ & 1.8 & 0.8 & 1.4 \\
\hline 1-O-Caffeoylquinic acid & $0.3^{*}$ & $3.6^{*}$ & $0.3^{*}$ & $0.3 *$ & 0.7 \\
\hline c-3-O-Caffeoylquinic acid & 0.3 & 15.2 & $0.5^{*}$ & 0.7 & 1.0 \\
\hline t- 3-O-Caffeoylquinic acid & 0.2 & 7.2 & $0.4 *$ & 0.6 & 1.0 \\
\hline Catechol & $4.7 *$ & $6.5^{*}$ & 0.3 & $0.4 *$ & 0.5 \\
\hline Citric acid & $0.2 *$ & $0.2 *$ & 0.2 & 0.3 & 0.8 \\
\hline 2,5-Dihydroxybenzoic acid & $3.4 *$ & 3.3 & 0.6 & $0.6 *$ & 0.9 \\
\hline 3,4-Dihydroxybenzoic acid & 4.4 & 1.8 & $0.4 *$ & $0.5^{*}$ & 0.7 \\
\hline Fructose & 0.4 & 0.3 & $0.2 *$ & $0.4 *$ & $0.6 *$ \\
\hline Galactose & 1.1 & 0.4 & $0.2 *$ & $0.4 *$ & $0.5^{*}$ \\
\hline Galactosylpyranosylglycerol & 1.3 & 0.9 & $0.4 *$ & $0.5 *$ & 0.8 \\
\hline Gentisic acid-5-O-glucoside & 2.2 & ND & 0.6 & 0.7 & 1.2 \\
\hline Glucose & 0.5 & $0.3 *$ & $0.3 *$ & $0.4 *$ & $0.6^{*}$ \\
\hline Glycerol & $3.2 *$ & 4.4 & 0.5 & 0.7 & 1.1 \\
\hline Hydroquinone & $97.9 *$ & 9.7 & 1.1 & 1.2 & 1.3 \\
\hline 3-Hydroxybenzoic acid & $7.2 *$ & $5.7 *$ & 0.7 & 0.8 & 0.9 \\
\hline 4-Hydroxybenzoic acid & $4.2 *$ & 1.3 & $0.6^{*}$ & 0.8 & 1.0 \\
\hline$\alpha$-Linolenic acid & 2.0 & 2.3 & $0.5^{*}$ & $0.6^{*}$ & 0.9 \\
\hline Maleic acid & 0.7 & $2.6^{*}$ & $0.3 *$ & 0.6 & 0.7 \\
\hline Malic acid & 0.2 & $0.3 *$ & $0.2 *$ & 0.3 & 0.8 \\
\hline Mannitol & $2.3 *$ & 0.9 & 5.6 & 14.5 & 12.9 \\
\hline Oxalomalic acid & 1.1 & 1.6 & 0.3 & 0.5 & 1.1 \\
\hline Palmitic acid & $5.9 *$ & $4.1^{*}$ & $0.6^{*}$ & 0.8 & 1.0 \\
\hline Phosphate & 1.2 & 0.3 & 0.3 & 0.3 & 1.8 \\
\hline Pyruvic acid & $6.6^{*}$ & $3.9 *$ & 0.8 & 0.7 & 1.1 \\
\hline Quinic acid & 0.3 & 1.5 & $0.2 *$ & 0.7 & 0.6 \\
\hline Salicin & 0.6 & $3.9^{*}$ & 2.6 & 0.9 & 4.3 \\
\hline Salicortin & 0.2 & 3.9 & 0.7 & 0.5 & 3.5 \\
\hline$\alpha$-Salicyloylsalicin & 0.3 & 8.7 & 0.5 & 0.9 & 2.4 \\
\hline$\beta$-Sitosterol & 1.9 & 0.6 & $0.5^{*}$ & $0.5^{*}$ & 0.8 \\
\hline Stearic acid & 8.9 & 5.2 & 0.8 & 0.7 & 1.0 \\
\hline Sucrose & 0.3 & 1.1 & 0.8 & $1.3^{*}$ & 1.1 \\
\hline Syringic acid & $6.4 *$ & 1.0 & 0.7 & 0.7 & 1.9 \\
\hline Trehalose & $39.7 *$ & $25.0 *$ & 1.2 & $0.6^{*}$ & 1.0 \\
\hline Trichocarpin & 0.3 & ND & 0.5 & 0.4 & 1.6 \\
\hline 1,3,5-Trihydroxybenzene & $3.1 *$ & $6.1 *$ & 0.7 & 1.1 & 1.0 \\
\hline Tryptophan & 0.4 & $5.6^{*}$ & 0.1 & 0.1 & 0.3 \\
\hline
\end{tabular}

a Symbols: $\infty$ indicates that there was no detectable metabolite in control tissues and that there were high amounts of detectable metabolite in colonized tissues; * indicates $P<0.05$ (Student's $t$ test test); ND = not detected. 
with an atypical increase in succinic acid and fumaric acid despite the decline of other TCA cycle organic acids, suggested that one of the largest impacts of the colonization process was the redirection of carbon flux to the TCA cycle via the benzoate degradation pathway. To further confirm this hypothesis, we mapped the metabolite fold-change responses from 12week ECM root tips onto the Kyoto Encyclopedia of Genes and Genomes benzoate degradation pathway (red values; Fig. 2). We found that all measured metabolites leading to the synthesis of arbutin and succinic acid were increased in ECM root tips compared with control tissues.

In order to understand which of the partners may be driving the accumulation of these compounds, we analyzed the foldchange in gene expression of the annotated enzymes controlling metabolic flux in this pathway from both the $P$. trichocarpa (green values) and L. bicolor (brown values) partners at 2, 4, and 12 weeks post interaction (Fig. 2). In the metabolism of benzoate to succinic acid, L. bicolor showed consistent upregulation of key enzymes to create catechol through to succinyl-CoA at all three time points analyzed. The regulated enzymes included catechol 1,2-dioxygenase (EC: 1.13.11.1), 3-oxoadipate CoA-transferase, $\alpha$ subunit (EC: 2.8.3.6), and especially acetyl-CoA acyltransferase (EC: 2.3.1.16). Of the annotated enzymes within this pathway encoded by $P$. trichocarpa, only dihydroxycyclohexadiene carboxylate dehydrogenase (EC: 1.3.1.25) showed a large fold-increase in transcript abundance at 4 weeks post contact. Therefore, based on current annotated gene function, L. bicolor appears to be the primary driver of the accumulation of succinic acid.

L. bicolor also appears to be the primary driver in the early stages of the conversion of benzoate to arbutin because benzoate 4-monooxygenase (EC: 1.14.13.12) is highly upregulated in fungal tissues. From this point on in the arbutin metabolic pathway, however, it is very difficult to determine which organism is responsible for producing the enzymes that drive these pathways. This is due to a lack of annotations for key enzymes such as gentisate decarboxylase (EC: 4.1.1.62) and 4-hydroxybenzoate 1-hydroxylase (EC: 1.14.13.64) in L. bicolor and $P$. trichocarpa. At the metabolic level, however, both the pathways to arbutin from 3- and 4-hydroxybenzoic acid appear to be important because these key precursors are found to be 7.2- and 4.2-fold increased, respectively, in ECM root tips. It is interesting to note that the upregulation of the benzoate degradation pathway by L. bicolor was also correlated with the decline in two benzyl alcohol-containing phenolic glycosides, including trichocarpin by week 12 and the partially identified metabolite 13.97 benzylglycoside at as early as 2 weeks.

\section{Incompatible interaction: metabolic profiles associated with a recalcitrant host.}

As detailed in the first section of the Results, $P$. deltoides is a recalcitrant host that forms very few mature ECM root tips (Fig. 1). The recalcitrant nature of this host tree is genetic in its basis (Labbé et al. 2011); therefore, we wanted to characterize the differences in metabolic behavior between $L$. bicolor (WT)-P. deltoides ECM root tips and L. bicolor (WT)-P. trichocarpa ECM root tips. At 12 weeks after fungal inoculation, L. bicolor (WT)-P. deltoides mycorrhizal roots had increases in a number of the same metabolites as colonized $P$. trichocarpa roots but the magnitude of the increase was generally lower. For example, arbutin levels in mycorrhizal roots of $P$. deltoides after 12 weeks were $13 \times$ less than levels found in $P$. trichocarpa ECM root tips, whereas its aglycone (hydroquinone; 1,4-dihydroxybenzene) was $10 \times$ lower in $P$. deltoides ECM root tips. Although the symbiotic tissues of both Populus spp. accumulated trehalose in response to mycorrhization, the concentration was higher in $P$. trichocarpa at week 12 than in
$P$. deltoides, which may be due to higher amounts of fungal mycelia or a higher rate of sugar transfer within the L. bicolor (WT) $-P$. trichocarpa mycorrhizal root tips (Table 2). In addition to these large changes, there was a series of smaller increases in a number of aromatic metabolites, including 3-hydroxybenzoic acid, phloroglucinol (1,3,5-trihydroxybenzene), benzoic acid, and catechol, that were significant $(P \leq 0.05)$ in both Populus spp. (Table 2). Given that microbes can decarboxylate salicylic acid to produce catechol (Walker and Evans 1952), it should be noted that salicylic acid itself was unaffected by colonization in either Populus spp. (Table 2).

Other notable differences in the species' responses included a fourfold increase in salicin in P. deltoides, a Populus sp. defense metabolite, with no increase evident in $P$. trichocarpa at the same 12-week time point (Table 2). In contrast, trichocarpin, another higher-order salicylate that occurs in $P$. trichocarpa but not $P$. deltoides, was repressed compared with nonmycorrhizal tissues. Other higher-order salicylates, including salicortin and $\alpha$-salicyloylsalicin, remained at high concentrations in $P$. deltoides. There was a differential response between species in 1-O-caffeoylquinic acid that declined in $P$. trichocarpa but remained the same or was higher in $P$. deltoides. One additional contrast of note, given its importance as a substrate for indole-conjugates that are known defense response metabolites, is the 5.6-fold accumulation of tryptophan in $P$. deltoides that was not evident in P. trichocarpa (Table 2).

\section{Incompatible interaction: metabolic profiles associated with $\boldsymbol{L}$. bicolor mutants unable to colonize $P$. trichocarpa.}

In addition to determining how the metabolome of ECM root tips was altered when the host plant was recalcitrant to colonization, we also determined how the metabolome of plant tissue changed after 12 weeks of contact with a fungus that was unable to colonize a natural host plant. To do this, we used three mutant lines of $L$. bicolor that are unable to fully colonize the roots of $P$. trichocarpa (L. bicolor missp7 RNAi (RNA interference) lines CL03, CL09, and CL19; Fig. 1B) (Plett et al. 2011). In general, the metabolite responses associated with normal symbiotic association were still present in roots in contact with L. bicolor missp 7 mutants (Table 2) but, as in the $L$. bicolor (WT)-P. deltoides interaction, the fold-changes of these metabolites were greatly diminished. The $254 \mathrm{~m} / \mathrm{z}$ (likely hydroquinone) conjugates and the two most abundant $244 \mathrm{~m} / \mathrm{z}$ alkaloids that were present in the compatible L. bicolor (WT)$P$. trichocarpa interaction were present even with the $L$. $b i$ color missp 7 mutants. However, the large 173-fold increase in arbutin observed with symbiosis between L. bicolor (WT) and P. trichocarpa was no longer significantly regulated (Table 2). Furthermore, the fivefold increase in hydroquinone, its aglycone, was not evident in L. bicolor missp7 mutant ECM root tips. For the metabolites that tended to decline in the normal

Table 3. Laccaria bicolor is able to metabolize Populus metabolites with differing degrees of efficacy

\begin{tabular}{lcc}
\hline Added metabolite & Measured metabolite & Laccaria $^{\text {bicolor }}$ \\
\hline Arbutin & Arbutin & $0.6^{*}$ \\
& Hydroquinone & $3.4^{*}$ \\
Hydroquinone & Hydroquinone & $0.1^{*}$ \\
Salicin & Salicin & $0.2^{*}$ \\
& Salicylic acid & $94.8^{*}$ \\
Chlorogenic acid & Salicyl alcohol & 10.7 \\
& Chlorogenic acid & 1.4 \\
& Quinic acid & $5.0^{*}$ \\
& Caffeic acid & 0.7
\end{tabular}

${ }^{a}$ Values reported are fold-change in metabolite level compared with supplemented medium without fungal presence. Asterisk (*) indicates $P<$ 0.05 (Student's $t$ test). 
mycorrhizal condition, including the soluble sugars glucose, fructose, and galactose and hydroxycinnamate-quinate esters, those declines were greater and statistically significant in fine roots colonized by the defective missp 7 mutants, particularly CL09 and CL19 (Table 2). The fungal sugar trehalose, whose accumulation is normally associated with functioning mycorrhizal root tips, was not significantly regulated in ECM roots associated with the L. bicolor missp 7 mutants, except in CL19. The lack of trehalose in these samples would suggest that these are not functional ECM root tips and also may be due to lower levels of fungal tissues in the roots sampled.

\section{L. bicolor can degrade certain plant secondary metabolites.}

Given the high levels of accumulation of metabolites known to be secreted and which have been implicated in plant defense (e.g., arbutin, hydroquinone, chlorogenic acid, and salicin), we determined whether L. bicolor was able to degrade them. Changes in the concentration of arbutin in the media in which
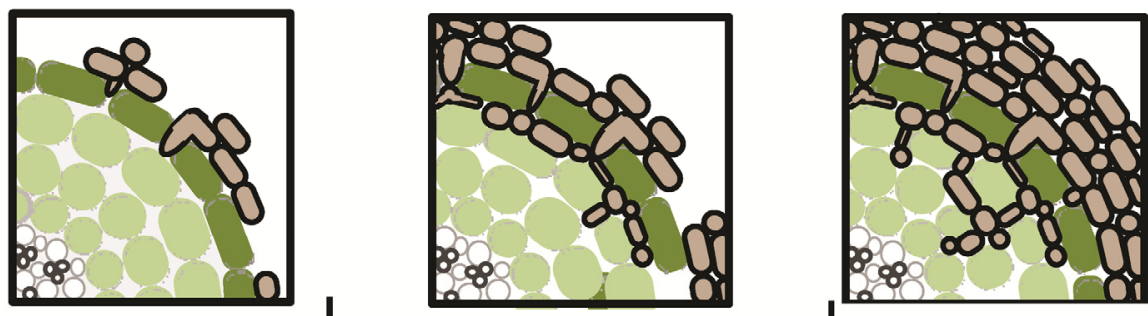

Initial Fungal:Plant Contact. Mantle Formation, Hartig Net Initiation, Mature ECM Root Tip

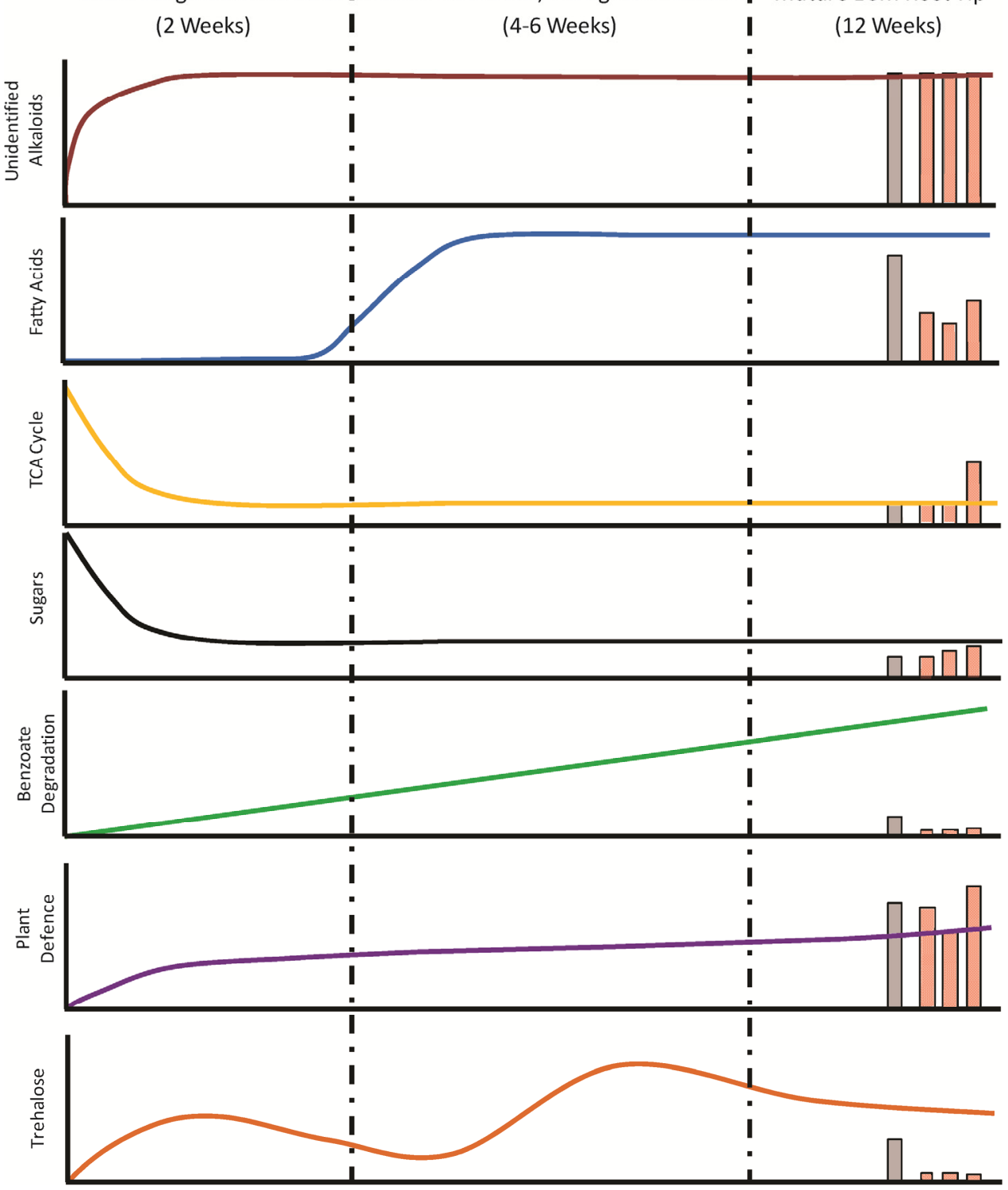

Fig. 3. Schematic representation of major trends observed in different metabolic pathways. Line graphs represent generalized trends in combined metabolic fluxes in different pathways (e.g., trichloroacetic acid [TCA] cycle) or for different metabolites (e.g., trehalose) over the course of colonization. Graphic representation of a transverse cross section of a root is shown above each time point to demonstrate the corresponding stage of colonization. Within the 12-week frame, gray bars represent the equivalent metabolic accumulation in mature ectomycorrhizal (ECM) root tips of Laccaria bicolor (WT)-Populus deltoids while the pink bars represent the relative metabolic accumulation of the three L. bicolor missp7 RNA interference transgenic lines (in the order of Cl09, $\mathrm{Cl19}$, and $\mathrm{Cl03}$ ). 
L. bicolor was grown were modest (64\% decline versus the no microbe control) but led to a threefold accumulation of its aglycone, hydroquinone, suggesting arbutin cleavage by this species (Table 3). Large declines in hydroquinone following its addition to the media were observed in L. bicolor $(0.1 \times$ of its original concentration). Following salicin addition, L. bicolor metabolized it to 0.2 -fold its original level. The primary salicin cleavage product, salicyl alcohol, was 11-fold higher in the medium on which the Laccaria sp. was grown, whereas an oxidation product of salicyl alcohol, salicylic acid, exhibited an even more dramatic increase (95-fold) in the Laccaria sp. (Table 3). Application of chlorogenic acid to the media resulted in a fivefold accumulation of the cleavage product quinic acid, whereas the aglycone cleavage product, caffeic acid, was not significantly affected. Therefore, L. bicolor has a differential ability to metabolize plant-derived compounds associated with defense.

\section{DISCUSSION}

Forest trees interact with and depend upon different organisms that are found in soils (Buée et al. 2009; Mendes et al. 2013; Philippot et al. 2013; Turnau et al. 1996). Building upon previous studies, we sought to understand how metabolism was altered during a compatible mutualistic interaction between $P$. trichocarpa and the ECM fungus L. bicolor. Functionally, the general responses associated with ECM colonization of Populus tissues included a decrease in the organic acids early in the TCA cycle (citric acid and aconitic acid) but increased concentrations in those later in the TCA cycle, including succinic acid and fumaric acid, and an increase in metabolites related to fatty acid metabolism (Table 1; Fig. 3). Increased demand by the microbial symbiont for organic substrates is likely the cause for the observed decline in early TCA cycle organic acids and was an anticipated observation due to previous research characterizing the primary metabolism of the $\mathrm{Eu}$ calyptus-Pisolithus interaction (Martin et al. 1998; Schaeffer et al. 1995), the predicted metabolic pathway fluxes based on transcriptomic studies of the Laccaria-Populus interaction (Larsen et al. 2011), and given that the ECM fungi are very reliant on their host for carbon-rich compounds due to the reduced genomic potential for cleaving complex sugars and carbohydrates (Martin et al. 2008; Nehls et al. 2000, 2001; Weise et al. 2000). It is interesting to note the differences between the symbiotic metabolome of poplar roots colonized by ECM fungi such as L. bicolor in comparison with Medicago truncatula roots colonized by the arbuscular mycorrhizal (AM) fungus Rhizophagus irregulare (formerly Glomus intraradices; Schliemann et al. 2008). Although some aspects of both ECM and AM symbioses are similar (e.g., trehalose accumulation), the general metabolic response of poplar is very different. The decline or absence of significant regulation of sugars found in ECM-colonized root tips (e.g. glucose, fructose, and sucrose) was not found in AM-colonized roots, whereas all of the fatty acids we found to be increased in ECM-colonized roots are not regulated in AM-colonized roots (Fester et al. 2011; Schaeffer et al. 1997). These differences could be due to the very different architecture of the symbiotic tissues created to house these two classes of mutualistic fungi and the very strong carbon sink provided by large bulk of the extramatrical ECM fungal hyphae (Schaeffer et al. 1997), or to the different physiologies of the two plant hosts. Because Populus spp. can host both AM and ECM fungi, it would be interesting to compare the host symbiotic response to both of these fungi under similar conditions. Such a multiple interaction study would more closely mimic the real-world state, where a host plant and its resulting symbiotic metabolome will reflect the net effect of a large number of simultaneous mutualistic associations.

Upregulation of the benzoate degradation pathway by $L . b i$ color explains many of the largest metabolomic responses observed as the symbiosis is established between L. bicolor (WT) and P. trichocarpa (Table 1; Fig. 3). This includes the decline in benzyl-alcohol-containing phenolic glycosides and the accumulation of benzoic acid and many hydroxylated benzoic acid metabolites that, in turn, result in the accumulation of succinic acid. Succinic acid then feeds into the citric acid cycle, which is depleted by the symbiosis. The finding that benzoate degradation is one of the key metabolic pathways in ECM root tips is intriguing because such pathways are normally found to be induced in bacterial consortia, such as in syntrophic consortia (Warikoo et al. 1996), or during the primary stages of biofilm formation (Karthikeyan et al. 1999) for the primary purpose of xenobiotic detoxification. Benzoate detoxification has also been implicated as an important step in the symbiotic relationship between nematodes and their gut microbiome (Cheng et al. 2013), between Nasutitermes spp. and their gut fungal communities (Liu et al. 2013), and in the interaction between legumes and bradyrhizobia (Mornico et al. 2011). Therefore, the induction of the benzoate degradation pathway in the L. bicolor-P. trichocarpa mutualism appears to be a common theme in multispecies interactions although, given the current data, we cannot determine whether the role of this pathway is to detoxify defensive xenobiotics or if this pathway serves to generate later TCA cycle organic acids or fill another, unidentified, role.

The second aim of this study was to contrast the metabolomes of ECM root tips in compatible and incompatible interactions. In the interaction between plants and nematodes, susceptible plants are characterized by a larger number of metabolic changes and a greater amplitude of metabolic fluxes, whereas resistant plant hosts were characterized by a repressed metabolic response (Afzal et al. 2009). In a comparison of compatible and incompatible interactions between Magnaporthe grisea and Oryza sativa, there was a great deal of overlap observed in the metabolic response of the plant (Jones et al. 2011), with the major difference found between compatible and incompatible reactions being the suppression of energy metabolism (e.g., citric acid cycle) and a higher alanine leaf content in compatible interactions. Additionally, as found in plant hosts resistant to nematode infection, incompatible hosts of $O$. sativa were characterized by an overall unresponsive metabolism when challenged with $M$. grisea. Our comparison of compatible and incompatible reactions to L. bicolor using both different host plants ( $P$. trichocarpa versus $P$. deltoides) and mutant L. bicolor strains (WT versus Missp7 RNAi lines) bear some similarities to these previous findings. In both cases, an incompatible relationship was characterized by a reduction in the number of significantly regulated metabolites in plant tissues in contact with fungal hyphae. Instead of a large increase in alanine content as observed by Jones and colleagues (2011) during incompatible relationships, we found, in the interaction between $L$. bicolor and $P$. deltoides, an increase in tryptophan-a precursor to many defense metabolites. This led us to consider whether incompatible relationships were characterized by a higher accumulation of metabolites associated with defense. Whereas a number of the metabolites associated with defense against insects and biotrophic pathogens were induced in the roots of both host plants undergoing colonization by $L$. bicolor (Brechenmacher et al. 2010; Hopkins et al. 2009; van Dam et al. 2009), our study indicates that $P$. trichocarpa is not more easily colonized due to a lack of defensive metabolites but, rather, an inability to maintain defense metabolites. This is in contrast with $P$. deltoides, which maintained elevated de- 
fense compounds at week 12. For example, few defense compounds typically associated with Populus spp. were elevated at week 12 in $P$. trichocarpa but $P$. deltoides roots in contact with L. bicolor for 12 weeks maintained a number of elevated defense metabolites, including salicin, 1-O-caffeoyl-quinic acid, and phloroglucinol (1,3,5-trihydroxybenzene). Therefore, rather than identifying specific metabolites that are responsible for the different colonization rates between the compatible and incompatible relationships studied here, our results would suggest that two of the main drivers that define compatible versus incompatible interactions lie in both the diversity and the halflife of defensive metabolites produced by the host as well as the general metabolic responsiveness of roots in the primary stages of colonization.

Our present study, therefore, has demonstrated that the colonization process of poplar roots by the ECM fungus L. bicolor dramatically alters the metabolic processing within the symbiotic ECM root tissues. These changes to the symbiotic metabolome are not static but alter throughout the colonization process. Therefore, based on our results, it is likely that, for the intimate mutualistic ECM relationship to proceed, host defenses must be suppressed or moderated, allowing for the fungus to integrate into the root tissues. The process of fungal integration within plant tissues then causes the observed deep modification to a number of host metabolic pathways. Conversely, should these host defenses not be moderated, as we observed in incompatible interactions, the fungus will be repulsed from the host root system and the metabolic changes observed in compatible interactions will never be induced. It will be interesting in the coming years to determine how ECM fungi are able to manipulate plant defense metabolic pathways to their favor in compatible interactions.

\section{MATERIALS AND METHODS}

\section{Mycorrhiza formation time course.}

Mycorrhiza formation trials were carried out as described by Plett and associates (2011) in a climate-controlled greenhouse in a randomized block pattern. Unless otherwise noted, samples were taken from three independent plants for each data point. In short, cuttings of either $P$. trichocarpa (Washington clone 101-74) or P. deltoides (Illinois clone 73028-62) were prerooted in nutrient hydroponic solution for 2 weeks before being potted in Terra-Green (a calcined attapulgite clay supplied by Turf-Pro; Brenntag SA, Toul, France) dosed with $10 \%$ L. bicolor $\mathrm{S} 238 \mathrm{~N}$ or L. bicolor missp7 RNAi independent transgenic lines CL03, CL09, or CL19 as a fungal inoculums (Plett et al. 2011). Control plants were grown in pure, sterile TerraGreen. For P. trichocarpa, a minimum of 20-mg samples were taken at 2-week intervals; short roots colonized by fungal mycelia (mycorrhizal root tips) were sampled and flash frozen in liquid nitrogen. Fine lateral roots of plants grown without fungal inocula were used as a control. For P. trichocarpa in association with L. bicolor missp7 RNAi lines (CL03, CL09, and CL19) versus WT L. bicolor $\mathrm{S} 238 \mathrm{~N}$ or P. trichocarpacolonized roots compared with $P$. deltoides-colonized roots, only 12-week-old mycorrhizal root tips were collected for analysis, with three replicate samples per association. Percent mycorrhization was calculated by averaging the percentage of lateral roots colonized in three random root samplings per tree (three trees harvested per condition and a total of 900 lateral roots counted). In order to determine the depth of the Hartig net, colonized roots were fixed in $4 \%$ paraformaldehyde in $1 \times$ phosphate-buffered saline (PBS) $\left(\mathrm{pH} \mathrm{7.4)}\right.$ for $24 \mathrm{~h}$ at $4{ }^{\circ} \mathrm{C}$. Prior to inclusion in $6 \%$ agarose, fixed roots were washed three times for 15 min each in $1 \times$ PBS ( $\mathrm{pH} 7.4$ ). Embedded roots were then sectioned using a Lecia VT1200S vibratome. Only $30-\mu \mathrm{m}$ sections from the middle of the colonized root were taken and then stained in 1\% UVtex and observed using a BioRad Rainbow confocal scanning laser microscope (Nikon-BioRad, Marnes-la-Coquette, France). The depth of penetration was determined using ImageJ analysis of our microscopic images. Data presented in this article for the development of the Hartig net are the average of at least three biological replicates.

\section{Growth of fungal material.}

All fungal strains (L. bicolor [WT], L. bicolor missp7 CL03, L. bicolor missp7 CL09, and L. bicolor missp7 CL19) were maintained on potato dextrose agar (PDA) at $25^{\circ} \mathrm{C}$ in the dark. To test the growth effect of arbutin, hydroquinone, chlorogenic acid, or salicin, 4-mm plugs of actively growing $L$. bicolor (WT) mycelia were taken and used to inoculate PDA medium supplemented with of arbutin, hydroquinone, chlorogenic acid, or salicin (Supplementary Table S1) or water (control) and allowed to grow at $25^{\circ} \mathrm{C}$ for 20 days. Four biological replicates were performed.

\section{Metabolite extraction and analysis.}

Between 20 and $100 \mathrm{mg}$ fresh weight of sample was ground to a fine powder in liquid nitrogen, weighed, and then transferred to an acid-washed glass scintillation vial containing 2.5 $\mathrm{ml}$ of $80 \%$ ethanol (aqueous). Sorbitol $(75 \mu \mathrm{l}$ of a $1 \mathrm{mg} / \mathrm{ml}$ aqueous solution) was carefully added before extraction as an internal standard to correct for differences in extraction efficiency. The samples were extracted overnight and the solvent pipetted off into another vial the next day. The pellet was reextracted with another $2.5 \mathrm{ml}$ of $80 \%$ ethanol overnight and the supernatant combined with the prior extract. Of this solution 2 $\mathrm{ml}$ was dried using a nitrogen stream and stored at $-80^{\circ} \mathrm{C}$ until analysis. The samples were dissolved in $750 \mu \mathrm{l}$ of aqueous $80 \%$ ethanol, of which $500 \mu \mathrm{l}$ was dried in a nitrogen stream. Metabolite analyses using gas chromatography-mass spectrometry (GC-MS) were conducted, as described elsewhere (Tschaplinski et al. 2012). Briefly, dried extracts were dissolved in $500 \mu \mathrm{l}$ of silylation-grade acetonitrile followed by the addition of $500 \mu \mathrm{l}$ of $\mathrm{N}$-methyl-N-trimethylsilyltrifluoroacetamide with $1 \%$ trimethylchlorosilane (Thermo Scientific, Bellefonte, PA, U.S.A.), and samples then heated for $1 \mathrm{~h}$ at $70^{\circ} \mathrm{C}$ to generate trimethylsilyl (TMS) derivatives. After 2 days, 1- $\mu$ l aliquots were injected into an Agilent 5975C inert XL gas chromatograph-mass spectrometer (Agilent Technologies Inc., Santa Clara, CA, U.S.A.), fitted with an Rtx-5MS with Integra-Guard (5\% diphenyl/95\% dimethyl polysiloxane) capillary column ( $30 \mathrm{~m}$ by $250 \mu \mathrm{m}$ by $0.25 \mu \mathrm{m}$ film thickness) (Restek, Bellefonte, PA, U.S.A.). The standard quadrupole GC-MS was operated in the electron impact $(70 \mathrm{eV})$ ionization mode, in full scan (50 to $650 \mathrm{Da})$, under the operating conditions described earlier (Tschaplinski et al. 2012). A large user-created database $(>2,000$ spectra) of mass spectral electron ionization fragmentation patterns of TMS-derivatized compounds, as well as the Wiley Registry 8th Edition combined with NIST 05 mass spectral database, were used to identify the metabolites of interest to be quantified. Peaks were analyzed using a key selected ion, a characteristic $\mathrm{m} / \mathrm{z}$ fragment, rather than the total ion chromatogram, to minimize integrating co-eluting metabolites. The extracted peaks of known metabolites were scaled back up to the total ion current using predetermined scaling factors. The scaling factor for the internal standard (sorbitol) was used for unidentified metabolites. Unidentified metabolites were denoted by their retention time as well as key $\mathrm{m} / \mathrm{z}$ fragments. Peaks were quantified by area integration and were normalized to the quantity of the internal standard recovered, accounting for the volume of sample processed, derivatization volume, and injection volume. Three replicate plant samples 
were analyzed and the metabolite data were averaged by plant type and mycorrhizal status. The $P$ value was calculated using the Student's $t$ test and the comparison was between the means of sets of replicates for constituents for a given contrast, with the data expressed as fold-changes relative to a nonmycorrhizal condition (negative control). Various ratios of constituents will occasionally yield division by 0 , resulting in $\infty$, which is significant if it is a value divided by zero and not zero divided by zero.

\section{Transcript analysis.}

For RNA-Seq, four independent biological replicates of colonized and uncolonized tissues after 2, 4, and 12 weeks of contact between $P$. trichocarpa and L. bicolor as well as control mycelium were pooled and each sample was sequenced in an Illumina lane. Tissues were harvested from the same plants as for the metabolomic analysis and divided between RNA isolation and metabolite extraction to be the most comparable. Library construction and 36-bp single reads sequencing was performed by IGA Technology services (Udine, Italy). Raw reads were aligned to Ptricocarpa_210_transcripts taken from Phytozome v9.1 and corresponding to the $P$. trichocarpa genome v3 or to the Lacbi2 Gene catalog transcripts and corresponding to the L. bicolor genome v2 using CLC Genomics Workbench 6. For mapping, the minimum length fraction was 0.9 , the minimum similarity fraction 0.8 , and the maximum number of hits for a read was set to 10 . The unique and total mapped reads numbers for each transcript were determined, then normalized to reads per kilobase of exon model per million mapped reads. The complete expression dataset is available as series (accession number GSE54789) in the Gene Expression Omnibus database at the National Center for Biotechnology Information.

\section{ACKNOWLEDGMENTS}

This work was supported by the European Commission within the Project ENERGYPOPLAR (FP7-211917) and the ANR project FungEffector (to F. Martin). This research was also sponsored by the Genomic Science Program (Science Focus Area 'Plant:Microbe Interfaces'), U.S. Department of Energy, Office of Science, Biological and Environmental Research under the contract DE-AC05-00OR22725. F. Martin's research group is part of the Laboratory of Excellence ARBRE (ANR-11-LABX0002-01).

\section{LITERATURE CITED}

Afzal, A. J., Natarajan, A., Saini, N., Iqbal, M. J., Geisler, M., El Shemy, H. A., Mungur, R., Willmitzer, L., and Lightfoot, D. A. 2009. The nematode resistance allele at the rhg 1 locus alters the proteome and primary metabolism of soybean roots. Plant Physiol. 151:1264-1280.

Berger, S., Sinha, A. K., and Roitsch, T. 2007. Plant physiology meets phytopathology: Plant primary metabolism and plant-pathogen interactions. J. Exp. Bot. 58:4019-4026.

Brechenmacher, L., Lei, Z., Libault, M., Findley, S., Sugawara, M., Sadowsky, M. J., Sumner, L. W., and Stacey, G. 2010. Soybean metabolites regulated in root hairs in response to the symbiotic bacterium Bradyrhizobium japonicum. Plant Physiol. 153:1808-1822.

Buée, M., De Boer, W., Martin, F., Van Overbeek, L., and Jurkevitch, E. 2009. The rhizosphere zoo: An overview of plant-associated communities of microorganisms, including phages, bacteria, archaea, and fungi, and of some of their structuring factors. Plant Soil 321:189-212.

Burgess, T., Dell, B., and Malajczuk, N. 1996. In vitro synthesis of Pisolithus-Eucalyptus ectomycorrhizae: Synchronization of lateral tip emergence and ectomycorrhizal development. Mycorrhiza 6:189-196.

Ceccaroli, P., Buffalini, M., Saltarelli, R., Barbieri, E., Polidori, E., Ottonello, S., Kohler, A., Tisserant, E., Martin, F., and Stocchi, V. 2011. Genomic profiling of carbohydrate metabolism in the ectomycorrhizal fungus Tuber melanosporum. New Phytol. 189:751-764.

Cheng, X. Y., Tian, X. L., Wang, Y. S., Lin, R. M., Mao, Z. C., Chen, N., and Xie, B. Y. 2013. Metagenomic analysis of the pinewood nematode microbiome reveals a symbiotic relationship critical for xenobiotics degradation. Sci. Rep. 3:1869.
Deveau, A., Kohler, A., Frey-Klett, P., and Martin, F. 2008. The major pathways of carbohydrate metabolism in the ectomycorrhizal basidiomycete Laccaria bicolor S238N. New Phytol. 180:379-390.

Felten, J., Kohler, A., Morin, E., Bhalerae, R.P., Palme, K., Martin, F., Ditengou, F. A., and Legue, V. 2009. The ectomycorrhizal fungus Laccaria bicolor stimulates lateral root formation in poplar and Arabidopsis through auxin transport and signaling. Plant Physiol. 151:19912005.

Fester, T., Fetzer, I., Buchert, S., Lucas, R., Rillig, M. C., and Härtig, C. 2011. Towards a systemic metabolic signature of the arbuscular mycorrhizal interaction. Oecologia 167:913-924.

Finlay, R. D., Ek, H., Odham, G., and Soderstrom, B. 1988. Mycelial uptake, translocation and assimilation of nitrogen from $15 \mathrm{~N}$-labelled ammonium by Pinus sylvestris plants infected with four different ectomycorrhizal fungi. New Phytol. 110:59-66

Finlay, R. D., Ek, H., Odham, G. and Soderstrom, B. 1989. Uptake, translocation and assimilation of nitrogen from 15N-labelled ammonium and nitrate sources by intact ectomycorrhzial systems of Fagus sylvatica infected with Paxillus involutus. New Phytol. 113:47-55.

Gea, L., Normand, L., Vian, B., and Gay, G. 1994. Structural aspects of ectomycorrhiza of Pinus pinaster (Ait.) Sol. formed by an IAA overproducer mutant of Hebeloma cylindrosporum Romagnesi. New Phytol. 128:659-670.

Hopkins, R. J., van Dam, N. M., and van Loon, J. J. 2009. Role of glucosinolates in insect-plant relationships and multitrophic interactions. Annu. Rev. Entomol. 54:57-83.

Jones, O. A., Maguire, M. L., Griffin, J. L., Jung, Y. H., Shibato, J., Rakwal, R., Agrawal, G. K., and Jwa, N. S. 2011. Using metabolic profiling to assess plant-pathogen interactions: An example using rice (Oryza sativa) and the blast pathogen Magnaporthe grisea. Eur. J. Plant Pathol. 129:539-554.

Karthikeyan, S., Wolfaardt, G. M., Korber, D. R., and Caldwell, D. E. 1999. Identification of synergistic interactions among microorganisms in biofilms by digital image analysis. Int. Microbiol. 2:241-250.

Kottke, I., and Oberwinkler, F. 1987. The cellular structure of the Hartig net: Coenocytic and transfer-cell like organization. Nord. J. Bot. 7:8595.

Labbé, J., Jorge, V., Kohler, A., Vion, P., Marcais, B., Bastien, C., Tuskan, G. A., Martin. F., and Le Tacon, F. 2011. Identification of quantitative trait loci affecting ectomycorrhizal symbiosis in an interspecific F1 poplar cross and differential expression of genes in ectomycorrhizas of the two parents: Populus deltoides and Populus trichocarpa. Tree Genet. Gen. 7:617-627.

Larsen, P. E., Sreedasyam, A., Trivedi, G., Podila, G. K., Cseke, L. J., and Collart, F. R. 2011. Using next generation transcriptomic sequencing to predict an ectomycorrhizal metabolome. BMC Syst. Biol. 5:70.

Liu, N., Zhang, L., Zhou, H., Zhang, M., Yan, X., Wang, Q., Long, Y., Xie, L, Wang, S., Huang, Y., and Zhou, Z. 2013. Metagenomic Insights into Metabolic Capacities of the Gut Microbiota in a Fungus-Cultivating Termite (Odontotermes yunnanensis). PloS One 8:e69184.

Luo, Z. B., Janz, D., Jian, X., Gobel, C., Wildhagen, H., Tan, Y., Rennenberg, H., Feussner, I., and Polle, A. 2009. Upgrading root physiology for stress tolerance by ectomycorrhizas: Insights from metabolite and transcriptional profiling into reprogramming for stress anticipation. Plant Physiol. 151:1902-1917.

Martin, F., Boiffin, V., and Pfeffer, P. E. 1998. Carbohydrate and amino acid metabolism in the Eucalyptus globulus-Pisolithus tinctorius ectomycorrhiza during glucose utilization. Plant Physiol. 118:627-635.

Martin, F., Aerts, A., Ahrén, D., Brun, A., Danchin, E. G., Duchaussoy, F., Gibon, J., Kohler, A., Lindquist, E., Pereda, V., Salamov, A., Shapiro, H. J., Wuyts, J., Blaudez, D., Buée, M., Brokstein, P., Canbäck, B., Cohen, D., Courty, P. E., Coutinho, P. M., Delaruelle, C., Detter, J. C., Deveau, A., DiFazio, S., Duplessis, S., Fraissinet-Tachet, L., Lucic, E., FreyKlett, P., Fourrey, C., Feussner, I., Gay, G., Grimwood, J., Hoegger, P. J., Jain, P., Kilaru, S., Labbé, J., Lin, Y. C., Legué, V., Le Tacon, F., Marmeisse, R., Melayah, D., Montanini, B., Muratet, M., Nehls, U., Niculita-Hirzel, H., Oudot-Le Secq, M. P., Peter, M., Quesneville, H., Rajashekar, B., Reich, M., Rouhier, N., Schmutz, J., Yin, T., Chalot, M., Henrissat, B., Kües, U., Lucas, S., Van de Peer, Y., Podila, G. K., Polle, A., Pukkila, P. J., Richardson, P. M., Rouzé, P., Sanders, I. R., Stajich, J. E., Tunlid, A., Tuskan, G., and Grigoriev, I. V. 2008. The genome of Laccaria bicolor provides insights into mycorrhizal symbiosis. Nature 452:88-92.

Mendes, R., Garbeva, P., and Raaijmakers, J. M. 2013. The rhizosphere microbiome: Significance of plant beneficial, plant pathogenic and human pathogenic microorganisms. FEMS (Fed. Eur. Microbiol. Soc.) Microbiol. Rev. 37:634-663.

Molina, R. 1982. Use of the ectomycorrhizal fungus Laccaria laccata in forestry. I. Consistency between isolates in effective colonization of containerized conifer seedlings. Can. J. For. Res. 12:469-473. 
Mornico, D., Miché, L., Béna, G., Nouwen, N., Verméglio, A., Vallenet, D., Smith, A. A. T., Giraud, E., Medigue, C., and Moulin, L. 2011. Comparative genomics of Aeschynomene symbionts: Insights into the ecological lifestyle of nod-independent photosynthetic bradyrhizobia. Genes 3:35-61.

Nehls, U., Wiese, J., and Hampp, R. 2000. Cloning of a Picea abies monosaccharide transporter gene and expression-analysis in plant tissues and ectomycorrhizas. Trees 14:334-338.

Nehls, U., Mikolajewski, S., Magel, E., and Hampp, R. 2001. The role of carbohydrates in ectomycorrhizal functioning: Gene expression and metabolic control. New Phytol. 150:533-541.

Philippot, L., Raaijmakers, J. M., Lemanceau, P., and van der Putten, W. H. 2013. Going back to the roots: The microbial ecology of the rhizosphere. Nat. Rev. Microbiol. 11:789-799.

Plett, J. M., Kemppainen, M., Kale, S. D., Kohler, A., Legué, V., Brun, A., Tyler, B. M., Pardo, A. G., and Martin, F. 2011. A secreted effector protein of Laccaria bicolor is required for symbiosis development. Curr. Biol. 21:1197-1230.

Reich, M., Gobel, C., Kohler, A., Buée, M., Martin, F., Feussner, I., and Polle, A. 2009. Fatty acid metabolism in the ectomycorrhizal fungus Laccaria bicolor. New Phytol. 182:950-964.

Schaeffer, C., Wallenda, T., Guttenberger, M., and Hampp, R. 1995. Acid invertase in mycorrhizal and non-mycorrhizal roots in Norway spruce (Picea abies (L.) Karst.) seedlings. New Phytol. 129:417-424.

Schaeffer, C., Wallenda, T., Hampp, R., Salzer, P., and Hager, A. 1997. Carbon allocation in mycorrhizae. Pages 393-407 in: Trees-Contributions to Modern Tree Physiology. H. Rennenberg, W. Eschrich, and H. Ziegler, eds. Backhuys Publishers, Leiden, The Netherlands.

Schliemann, W., Ammer, C., and Strack, D. 2008. Metabolite profiling of mycorrhizal roots of Medicago truncatula. Phytochemistry 69:112-146.

Tschaplinski, T. J., Standaert, R. F., Engle, N. L., Martin, M. Z., Sangha, A. K., Parks, J. M., Smith, J. C., Samuel, R., Jiang, N., Pu, Y., Ragauskas, A. J., Hamilton, C. Y., Fu, C., Wang, Z.-Y., Davison, B. H., Dixon, R. A., and Mielenz, J. R. 2012. Down-regulation of the caffeic acid $O$-methyltransferase gene in switchgrass reveals a novel monolignol analog. Biotechnol. Biofuel 5:71.

Turnau, K., Kottke, I., and Dexheimer, J. 1996. Toxic element filtering in Rhizopogon roseolus/Pinus sylvestris mycorrhizas collected from calamine dumps. Mycol. Res. 100:16-22.

van Dam, N. M., Tytgat, T. O. G., and Kirkegaard, J. A. 2009. Root and shoot glucosinolates: A comparison of their diversity, function and interactions in natural and managed ecosystems. Phytochem. Rev. 8:171-186.

Walker, N., and Evans, W. C. 1952. Pathways in the metabolism of the mono-hydroxybenzoic acids by soil bacteria. Biochem. J. 52:xxiii.

Warikoo, V., McInerney, M. J., Robinson, J. A., and Suflita, J. M. 1996. Interspecies acetate transfer influences the extent of anaerobic benzoate degradation by syntrophic consortia. Appl. Environ. Microbiol. 62:26-32.

Wiese, J., Kleber, R., Hampp, R., and Nehls, U. 2000. Functional characterization of the Amanita muscaria monosaccharide transporter Ammst1. Plant Biol. 2:1-5.

\section{AUTHOR-RECOMMENDED INTERNET RESOURCES}

Phytozome Populus trichocarpa dataset: www.phytozome.net/poplar.php Joint Genome Instittue Lacbi2 Gene catalog: genome.jgi-psf.org/Lacbi2/Lacbi2.download.html 